\title{
EFECTO DE LA PRESIÓN AMBIENTAL EN LA EFECTIVIDAD DEL LANZAMIENTO AL ARO Y LA ANSIEDAD PREVIAA LA EJECUCIÓN EN BASQUETBOLISTAS PRINCIPIANTES Y AVANZADOS
}

\author{
Guadalupe Delgado Socatelli
}

\author{
Recibido I-XI-2002 • Aceptado 10-XII-2002
}

\begin{abstract}
Resumen: Con el objetivo de determinar si las condiciones de presión ambiental dadas por: el tiempo de ejecución, y la ausencia o presencia de público afectaban el rendimiento de 10 jugadores de baloncesto principiantes y 10 jugadores avanzados al ejecutar 10 tiros libres al aro lo cual permitiría conocer los cambios en la ansiedad grupal e individual de los participantes mediante la aplicación de los instrumentos POMS de McNair, Loor y Droppleman (1971) y el CSAI-2 de Martens y Vealey (1990).

El análisis estadístico se efectuó mediante la aplicación de un ANOVA de medidas repetidas para buscar diferencias entre los intentos según el tipo de presión. La determinación de las diferencias entre grupos, entre las mediciones, según el nivel de presión ambiental, y la interacción de la presión de los grupos, se hizo mediante la aplicación de un ANOVA Factorial Mixto.

Los resultados de la investigación permitieron corroborar la individualidad de las respuestas de la ansiedad ante distintas situaciones y la importancia de la personalización del afrontamiento para el manejo de la ansiedad competitiva.
\end{abstract}

Palabras clave: Ansiedad, Tensión, Presión Ambiental, Efectividad, Zona de Rendimiento Óptimo, Individualización.

\section{Introducción}

Los últimos años han visto florecer un marcado interés por el estudio de las relaciones que se establecen entre los niveles de ansiedad, tensión y el rendimiento deportivo. Sin embargo, pareciera que aún el progreso de la investigación y los acuerdos entre quienes la realizan se halla en sus albores.

Es importante que el desarrollo de los estudios se haga a la luz de una fundamentación teórica precisa y universalmente aceptada. De ahí que sea necesario partir de las premisas básicas.

En la presente investigación el interés se centró en la medición de la ansiedad previa a la ejecución de una prueba y los cambios en la conducta deportiva a partir de diferentes situaciones de presión ambiental que podrían variar o generar niveles de ansiedad diferentes en cada jugador según su nivel de experiencia o familiaridad con la destreza.

Antes de iniciar el análisis de los fundamentos teóricos establecidos a partir de las investigaciones, es importante anotar de acuerdo con Gill (1994), que la tensión y las emociones son mejor entendidas desde una perspectiva multidimensional, como parte de un proceso. Quien investiga se apoyó en las propuestas básicas del modelo de Lazarus de 1986, el cual hace referencia a 
que la ansiedad es un proceso multivariable que cambia permanentemente en el tiempo, y que existen una serie de factores externos e internos que son activados ante ciertas circunstancias y que en conjunto se manifiestan de diferentes formas. Por otra parte, agrega, lo que en una situación es considerado una variable dependiente en otra puede ser independiente, lo que reafirma su multivariabilidad y el que hay una sobre posición de emociones y tensión que deben estudiarse con mucha precisión (Gill, 1994).

Cuando se está en presencia de una situación que genera un desajuste o desbalance entre las demandas que establece el medio y la capacidad de respuesta, pueden obtenerse respuestas negativas o positivas dependiendo de la percepción de cada sujeto con respecto a la situación (Wrisberg, 1994).

A este nivel es importante entonces determinar o conocer el nivel específico de tensión al que un sujeto responde de una manera positiva o negativa, a lo cual debe agregarse también el tipo y calidad de las fuentes de tensión que provocan la conducta.

En los últimos años, autores como Gordin y Reardon (1995), Hanin (1995) y Unesthal (1995), han enfatizado sobre la importancia de que la ejecución de alto rendimiento se realice bajo ciertas condiciones psicológicas que unidas a las cualidades físicas, favorecen el máximo logro deportivo. Esto es, los atletas alcanzan un nivel y perfilan zonas de rendimiento caracterizadas por ciertos estados de tensión que les permiten rendir al máximo, cada una de estas difiere de un sujeto a otro, especialmente por la percepción de cada uno con respecto a los factores ambientales, los cuales, pueden ser los mismos o idénticos para todos.

La ansiedad óptima parece ser absolutamente individual y puede ser alta, baja o media, además la mejor ejecución parece estar relacionada con ciertas condiciones de ansiedad y tensión, si la ejecución se realiza fuera de esa zona óptima de rendimiento que combina ejecución, ansiedad y tensión, es posible que el resultado sea negativo, así, tareas iguales y diferentes requieren situaciones específicas óptimas para su ejecución (Hannin, 1995). Entrar en esa zona de "flujo" implica que el atleta ha logrado balancear los retos o amenazas con sus capacidades de modo que funciona óptimamente y rinde al máximo (Gordin y Reardon, 1995).

Lo anterior remite a que cada sujeto tiene un nivel específico de activación para enfrentar las situaciones normales de la vida diaria o algunas otras que implican cierto nivel de reto, esta activación está caracterizada, por respuestas físicas y psicológicas específicas únicas para cada sujeto.

Así, dada una serie de circunstancias ambientales y personales frente a una situación competitiva, se va a producir o presentar en el sujeto un determinado estado de ansiedad con el cual va a enfrentar el reto.

Wrisberg (1994), en su artículo The arousal perfomance relationship, hace referencia a la multidimensionalidad de la ansiedad con base en lo manifestado por Spielberg (1994) quien a partir de una serie de investigaciones, concluye que en cuanto a la ansiedad se deben identificar dos componentes iniciales: la ansiedad de estado y la de rasgo, esta última conceptualizada como una disposición innata o adquirida que predispone al sujeto a percibir un determinado rango de situaciones no amenazantes como tales, en tanto que la ansiedad de estado se conceptúa como una reacción de tensión emocional del instante frente a situaciones, de modo que se producen respuestas subjetivas, físicas o emotivas, conscientes e inconscientes asociadas o acompañadas por una activación del sistema nervioso frente a la situación.

Lo fundamental en este punto, independientemente de aspectos metamotivacionales y de la existencia de estados de orientación hacia metas u objetivos, es que la tensión y la ansiedad van a afectar de un modo u otro la conducta o respuestas de los sujetos frente a las demandas internas o externas, (Wrisberg, 1994), y que puede establecerse una relación entre los niveles de ansiedad y la ejecución tanto de modo 
nomotético (al nivel de grupo) como idiográfico por su individualidad, (Hannin, 1995) y que si bien pueden existir algunas congruencias en los modelos obtenidos, más bien es posible determinar que cada sujeto presenta una conducta que difiere de la media grupal que se obtenga, así, se hace difícil generalizar los resultados de las respuestas de un grupo a la conducta de cada uno de los sujetos.

Algunas investigaciones han intentado acercarse a la determinación de modelos de ansiedad y ejecución, pero Wrisberg (1994), concluye que la evidencia disponible sugiere que los deportistas:

- difieren respecto al rango de activación y el nivel óptimo de la misma,

- en algunos casos usan respuestas emotivas pre-competencia para mejorar su ejecución

- la ejecución óptima de la perfomance es sensible a los diferentes tipos de ajuste pre-competencia que se utilicen para lograr el nivel óptimo de activación.

Burton (1988), mediante el uso del CSAI-2 en un estudio con nadadores de estilo libre (considerado de complejidad media), determinó que se rendían mejor con bajos niveles de ansiedad somática en las pruebas de velocidad, mientras que en distancias mayores (mayor complejidad), el nivel óptimo de ansiedad somática era el adecuado, pero había poca influencia sobre la ansiedad cognitiva o la autoconfianza en ambas condiciones, sin embargo, pareciera que los autores afirman que parece existir algún tipo de ajuste de parte de los atletas a la complejidad de la tarea. En baloncesto parecen ser deseables niveles de activación mayores para la defensa en tanto que para los lanzamientos de tiro libre, pareciera más adecuado un nivel bajo (Wrisberg, 1994).

Sumado a la complejidad de la tarea, debe apuntarse que las condiciones situacionales podrían ser otro factor que se relaciona con la activación, la tensión, la ansiedad y la ejecución. Wrisberg (1994) en su revisión de literatura cita el caso de un estudio de Lowe (1973), quien halló que los beisbolistas de la Liga Menor bateaban mejor bajo condiciones de presión media que cuando esta era baja o alta. El mismo autor reporta los resultados de múltiples estudios en los que se manipulan situaciones de competencia y de laboratorio, de tiempo, de experiencia, y otros factores con respecto a la ansiedad, tensión y ejecución y concluye que aún deben considerarse muchas situaciones más para determinar los niveles óptimos de ansiedad en relación con la perfomance, especialmente por las diferencias halladas en el nivel de percepción del reto entre cada uno de los sujetos, lo cual nuevamente apoya lo planteado inicialmente respecto a que las medias grupales de rendimiento y ansiedad difieren respecto a las individuales.

Considerando las referencias mencionadas esta investigación buscó hacer una combinación de variables relacionadas con tiempo y presencia de público a fin de determinar si el manejo experimental de estas, podría afectar la eficacia en la ejecución de una destreza de baloncesto, el lanzamiento al aro, para lo cual se establecieron tres situaciones experimentales de ambiente con una muestra de dos grupos de sujetos: principiantes y avanzados en la destreza del baloncesto, también interesó determinar la similitud o diferencia de los patrones de ansiedad y efectividad de la ejecución con base en los promedios grupales obtenidos.

\section{Metodología}

\section{Sujetos}

Este estudio se realizó con una muestra de 19 estudiantes varones divididos en dos grupos experimentales, principiantes y avanzados en el dominio del baloncesto. El primer grupo estaba formado por 9 estudiantes matriculados en el curso 
de Actividad Deportiva de Baloncesto, nivel principiante, durante el I semestre de 1996 de la Universidad de Costa Rica y el segundo formado por 10 sujetos constituyó el grupo jugadores nivel avanzado, que formaban parte de los equipos de baloncesto del Instituto Tecnológico de Costa Rica, campeones nacionales de la Tercera y Cuarta División.

Los sujetos del grupo principiante nunca habían jugado baloncesto e inclusive algunos apenas iniciaban su aprendizaje. Sus edades promedio eran de 18.88 años con +4.34 sin experiencia formal en algún equipo de baloncesto.

Por su parte, los avanzados tenían un promedio de 17.88 años con +4.22 y contaban con un promedio de 6.44 años de experiencia en las ligas de baloncesto que incluían Juegos Deportivos Nacionales y los Campeonatos Nacionales Oficiales.

\section{Instrumentos de medición}

La información relativa a la efectividad en el lanzamiento al aro se recopiló mediante una hoja control grupal en la que se estipulaba el tratamiento del día, el nombre de cada sujeto y el número de encestes realizados en la sesión de acuerdo con el tratamiento correspondiente.

La determinación de las características de la prueba de lanzamiento al aro se hizo con base en un estudio piloto con 5 basquetbolistas principiantes y 5 avanzados, quienes debían ejecutar la prueba en el mayor y menor tiempo posible, de este modo se establecieron las tres condiciones de ejecución: la primera corresponde a la situación sin presión que debían hacer en 1:30 minutos y sin la presencia de espectadores, la segunda se asignó a la categoría presión media con 1:10 minutos con público silencioso en las gradas, finalmente a la categoría presión alta se le asignó 55 segundos y público que intervenía en favor y en contra de cada ejecución del sujeto.

La inclusión de público en tres condiciones diferentes, se efectuó con base en el criterio de quien investiga y a partir de los resultados que se habían obtenido en un estudio piloto no publicado con jugadores de un equipo de Fútbol de la I División en Costa Rica en 1995, quienes manifestaron que el público constituía una de las principales fuentes de estrés y les generaba mucha ansiedad durante los encuentros.

La medición de la ansiedad se realizó mediante la aplicación del Competitive State Anxiety Inventory 2, (CSAI-2), de Martens y Vealey (1990), que mide la ansiedad a partir de tres componentes diferentes a nivel somático, cognitivo y autoconfianza. También se utilizó el POMS de McNair, Lorr y Droppleman (1971) el cual mide los estados de ánimo, sin embargo para efectos de esta investigación se utilizó sólo el componente tensión el cual de acuerdo con la investigadora es el más directamente relacionado con la ansiedad.

Por otra parte se midió radialmente la frecuencia cardiaca de los sujetos a fin de contar con un indicador fisiológico de fácil medición directamente asociado con la ansiedad y de acuerdo con la experiencia de una investigación anterior realizada con un equipo de fútbol de la I División Nacional, (Delgado 1995, 1996).

\section{Procedimiento para la recopilación de la información}

La recopilación de los datos se efectuó de la siguiente manera:

- Cada grupo de sujetos efectuó por separado.

- Cada jugador debía ejecutar un total de 10 lanzamientos al aro desde la línea de tiro libre.

- La prueba iniciaba con el sujeto colocado en el centro de la cancha de baloncesto, a la señal se dirigía hacia cualquiera de las zonas de lanzamiento de tiros libres, tomaba uno de los 5 balones colocados al lado derecho de cada zona y lo lanzaba. Sin importar 
el resultado debía dirigirse de inmediato al otro tablero a ejecutar su siguiente lanzamiento y así sucesivamente hasta completar los 10 turnos establecidos.

- La ejecución de los 10 lanzamientos debía hacerse bajo tres condiciones diferentes de presión en razón del tiempo del que disponían para hacerlos y la presencia de observadores.

- Los sujetos ejecutaban las pruebas con 8 días de intervalo entre cada una.

- $\quad$ Antes de cada ejecución se le informó con detalle a cada sujeto las condiciones bajo las cuales ejecutarían la prueba, especialmente en cuanto al tiempo y la presencia o ausencia de espectadores.

- $\quad$ Posteriormente se medió la ansiedad mediante la aplicación de los instrumentos CSAI-2 y POMS.

- $\quad$ Finalmente se les tomó la frecuencia cardiaca radial.

\section{Análisis estadístico}

Se utilizó un ANOVA de una vía de medidas repetidas para determinar la significancia de las diferencias dentro de cada grupo según el tipo de presión en la variable efectividad y las psicológicas. Posteriormente se determinó el Tamaño del Efecto en cada variable y mediante el post hoc de Newmans Keuls se ubicó y cuantificó entre cuáles mediciones se daban las diferencias significativas por grupo que señalaba el Anova de una vía.

Para conocer las diferencias entre los grupos según el tipo de presión en la variable efectividad y las psicológicas se utilizó un ANOVA factorial mixto de $2 \times 3$.

Mediante la $r$ de Pearson se establecieron las correlaciones entre la variable efectividad y cada variable psicológica en cada uno de los grupos según el tipo de presión.

\section{Resultados}

En primera instancia se anotan los resultados de la estadística descriptiva en el que se resumen los promedios y desviaciones estandar de la efectividad y las variables psicológicas según el tipo de presión o tratamiento ejercido. En el Cuadro No. 1 se presentan los resultados de los promedios a partir de los cuales se realizaron los posteriores análisis estadísticos inferenciales.

Cuadro No. 1

Promedios y desviaciones estándar por grupo y variable según el tipo de presión

\begin{tabular}{|c|c|c|c|c|c|c|}
\hline \multirow[b]{3}{*}{ Efectividad } & \multicolumn{3}{|c|}{ Principiantes } & \multicolumn{3}{|c|}{ Avanzados } \\
\hline & \multirow{2}{*}{$\begin{array}{c}\text { Sin } \\
\text { presión }\end{array}$} & $\begin{array}{c}\text { Presión } \\
\text { media }\end{array}$ & $\begin{array}{c}\text { Presión } \\
\text { Alta }\end{array}$ & \multirow{2}{*}{$\begin{array}{c}\text { Sin } \\
\text { Presión }\end{array}$} & $\begin{array}{c}\text { Presión } \\
\text { media }\end{array}$ & $\begin{array}{c}\text { Presión } \\
\text { Alta }\end{array}$ \\
\hline & & \pm 2.12 & $2.44 \pm 1.66$ & & $4.50 \pm 1.77$ & $3 \pm 1.49$ \\
\hline $\begin{array}{l}\text { Ansiedad } \\
\text { congnitiva }\end{array}$ & $1.83 \pm .54$ & $1.77 \pm$ & $1.55 \pm .42$ & $2.04 \pm .44$ & $1.81 \pm$ & $1.80 \pm .45$ \\
\hline $\begin{array}{l}\text { Ansiedad } \\
\text { somática }\end{array}$ & $1.48 \pm .33$ & $1.28 \pm$ & $1.30 \pm .29$ & $1.66 \pm .57$ & $1.58 \pm$ & $1.38 \pm .32$ \\
\hline Autoconfianza & $2.82 \pm .38$ & $2.65 \pm$ & $2.61 \pm .62$ & $2.97 \pm .58$ & $3.14 \pm$ & $2.99 \pm 4$ \\
\hline $\begin{array}{l}\text { Frecuencia } \\
\text { cardiaca }\end{array}$ & $85.33 \pm 8.72$ & \pm 10.10 & $81.79 \pm 10.21$ & \pm 12.11 & \pm 11.34 & $74.40 \pm 10.36$ \\
\hline Tensión & $38.22 \pm 5.84$ & $36.88 \pm 4.70$ & \pm 4.35 & $36.5 \pm 4.39$ & \pm 3.77 & $34.66 \pm 4.16$ \\
\hline
\end{tabular}


Cuadro No. 2

Promedios marginales de cada grupo en las variables

\begin{tabular}{lcc} 
& Principiantes & Avanzados \\
& $\mathrm{X}$ & $\mathrm{X}$ \\
\hline & 2.85 & 4.23 \\
Efectividad & 1.72 & 1.88 \\
Ansiedad cognitiva & 1.54 \\
Ansiedad somática & 1.35 & 3.03 \\
Autoconfianza & 2.69 & 74.13 \\
Frecuencia cardíaca & 82.37 & 35.72 \\
Tensión & 36.70 & \\
\hline
\end{tabular}

Con base en ellos se pueden apreciar las diferencias entre los grupos de investigación respecto a la dimensión de las respuestas grupales.

Interesa complementar la anterior información con la del Cuadro No. 2, que resume los promedios marginales de cada variable por grupo sin importar el tipo de presión, obtenidos a partir del promedio de los tres promedios en cada variable de estudio.

El cuadro anterior en primera instancia permite demostrar que a partir de esos promedios ya los grupos mostraban diferencias iniciales. Estas diferencias permiten comparar los promedios marginales de cada grupo independientemente de la medición lo cual complementado con los promedios del Cuadro No. 1 favorece el análisis del comportamiento de todas las variables de estudio.

En cuanto a la efectividad es evidente que la diferencia podría explicarse en términos del dominio de la destreza. De igual forma se manifiestan los promedios de autoconfianza la cual es mayor en avanzados que en principiantes, la tensión que indica ser menor en los avanzados y finalmente frecuencia cardiaca que también es menor en los avanzados y podría explicarse de la misma forma.

El único aspecto que parece no se reporta de modo similar a los anteriores es el de la ansiedad somática.

Es a partir de la graficación de los resultados por grupo y, nivel de presión y variable que es posible observar las diferencias que se presentan de modo particular.

De este modo, bajo condiciones de presión media los principiantes obtienen los mejores promedios en tanto que los avanzados lo logran sin presión, sin embargo, el rendimiento de los avanzados siempre fue mayor que el de los principiantes en todas las mediciones y de modo total.

\section{Gráfico No. 1}

Efectividad por grupo según el tipo de presión

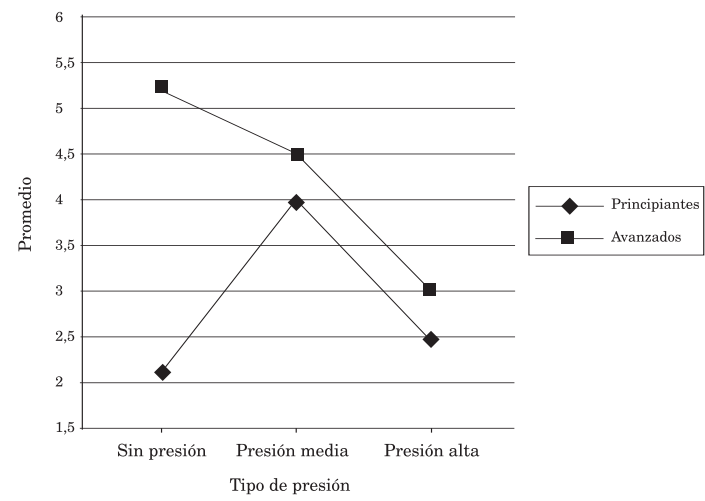

Debe agregarse que los jugadores avanzados muestran una relación lineal descendente de la primera a la tercera medición en tanto que los principiantes muestran una curva de $\mathrm{U}$ invertida en donde su mejor promedio se da en la segunda medición.

Los resultados de la aplicación del CSAI-2 y la frecuencia cardiaca en las tres 
mediciones se resumen a continuación y se apoyan con los cuadros anteriores y los gráficos respectivos.

En la ansiedad cognitiva esta tiende a ser mayor sin presión con respecto a las otras dos situaciones, sin embargo en los principiantes hay un descenso de 1.83 a 1.77 y luego a 1.55 en tanto que en los avanzados el descenso es mayor entre la primera y segunda medición, y no así entre esta y la tercera. En ambos casos, la ansiedad cognitiva es mayor sin presión y siempre es superior en los avanzados con respecto a los principiantes.

Gráfico No. 2

Ansiedad cognitiva por grupo según el tipo de presión

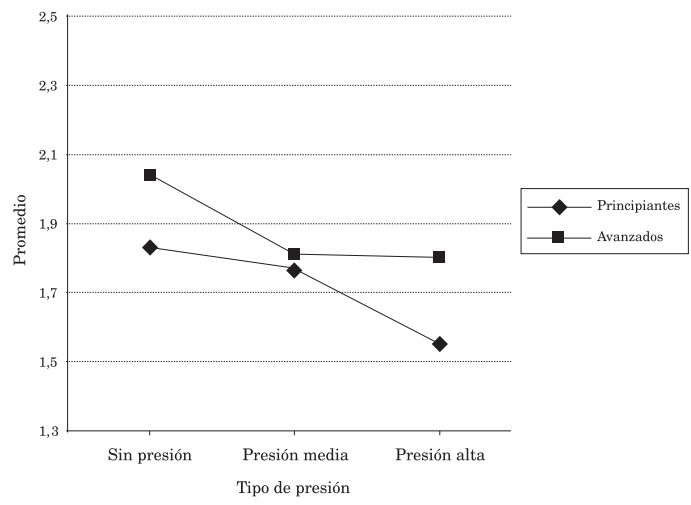

La ansiedad somática desciende bruscamente en los avanzados de 1.66 a 1.58 y 1.38 en tanto que en los principiantes se da un descenso importante entre la primera y segunda medición, (1.48 y 1.28), pero no así entre la segunda y tercera (1.28 y 1.30), además gráficamente se observa que la de los avanzados es siempre mayor que la de los principiantes.

La autoconfianza de los avanzados el mayor promedio se observa en la medición bajo presión media, (3.14), en tanto que 2.97 y 2.99 , promedios muy cercanos se presentan en la medición sin presión y presión alta respectivamente, esto permite afirmar que bajo condiciones de presión media la autoconfianza de este grupo tiende a ser mayor.
Gráfico No. 3

Ansiedad somática por grupos según el tipo de presión

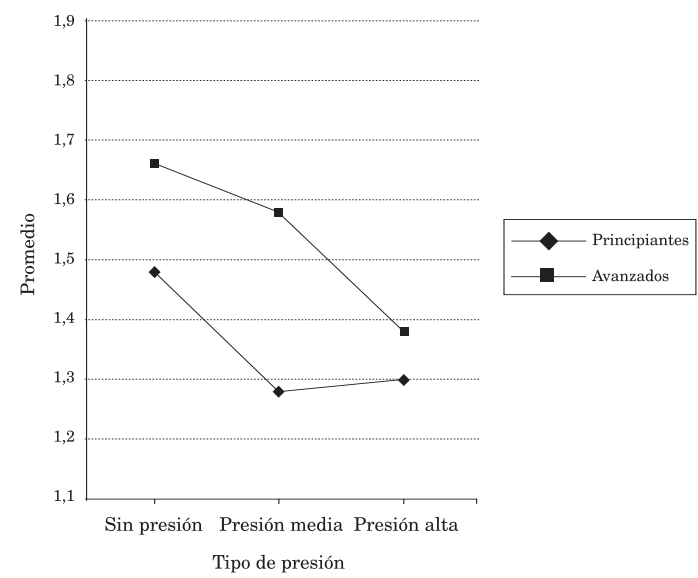

Los principiantes contrariamente, muestran una gradiente de descenso desde la primera a la tercera medición, 2.82 es el promedio mayor correspondiente a la ejecución sin presión, pasando luego a 2.65 y terminando con 2.61 , lo cual significa que la mayor autoconfianza la muestran sin presión. La mayor diferencia se da aquí entre la primera y segunda medición.

En el Gráfico No. 4 se apoya de nuevo que los avanzados tienen promedios mayores que los principiantes.

Gráfico No. 4

Autoconfianza por grupo según el tipo de presión

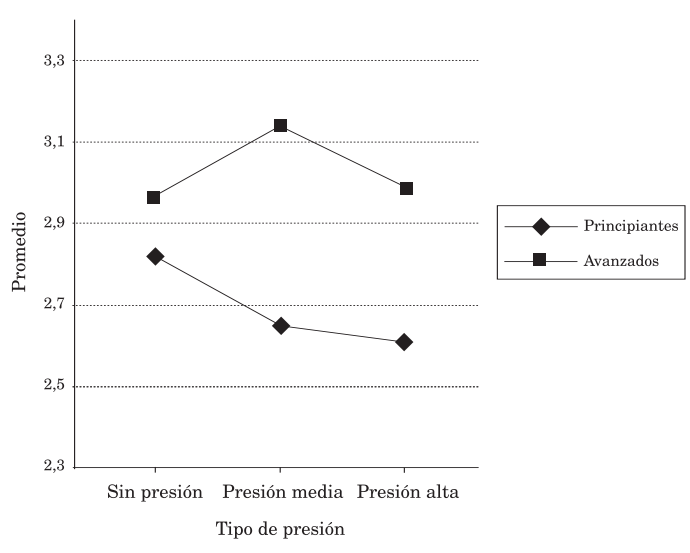


En la frecuencia cardiaca (Gráfico No. 5) de los avanzados se mantiene con promedios muy cercanos en las tres mediciones y siempre por debajo de los promedios de los principiantes, cuyo mayor promedio se muestra en la medición sin presión, 85.33 , la cual desciende a 80 con presión media y apenas sube a 81.79 en la tercera medición sin superar el promedio de la primera.

\section{Gráfico No. 5}

Frecuencia cardíaca por grupo según el tipo de presión

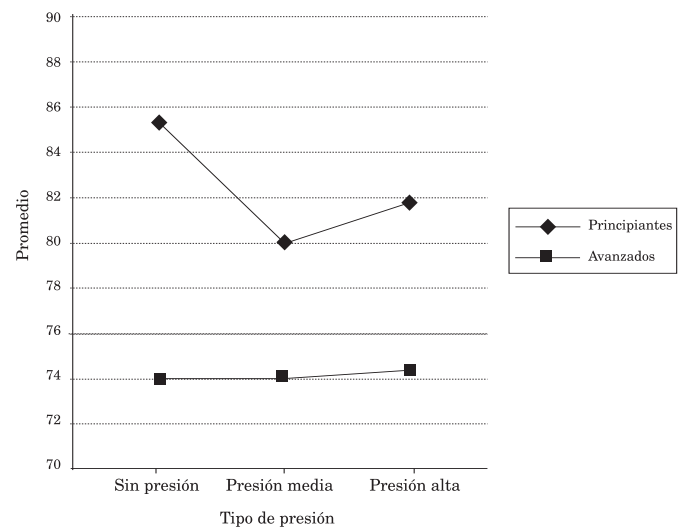

Al colocar en un sólo gráfico las mediciones de ansiedad cognitiva, somática y autoconfianza de cada grupo se observa que en ambos grupos la autoconfianza siempre estuvo por encima de las demás, seguida por la ansiedad cognitiva y al final la ansiedad somática.

En los principiantes siempre el promedio de la primera medición fue mayor que el de las otras dos situaciones; pero en los avanzados la situación es diferente. Sólo la autoconfianza muestra un aumento en la segunda medición y mantiene una forma de $\mathrm{U}$ invertida en tanto que la ansiedad cognitiva y la somática descienden gradualmente hacia la tercera medición.

El gráfico No. 7 muestra el caso de los avanzados. Se observa que sólo la autoconfianza muestra una clara forma de $\mathrm{U}$ invertida con poca diferencia entre la
Gráfico No. 6

Ansiedad del grupo de participantes según el tipo de presión

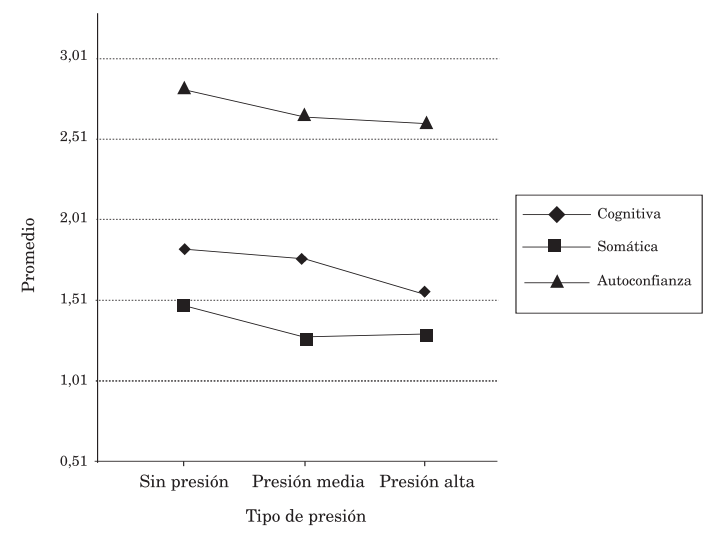

primera y tercera medición en tanto que la ansiedad cognitiva y la somática tiene promedios inferiores a los de la autoconfianza y siempre la primera medición al igual que el caso de los principiantes, es mayor que las otras dos mediciones.

Gráfico No. 7

Ansiedad del grupo de avanzados según el tipo de presión

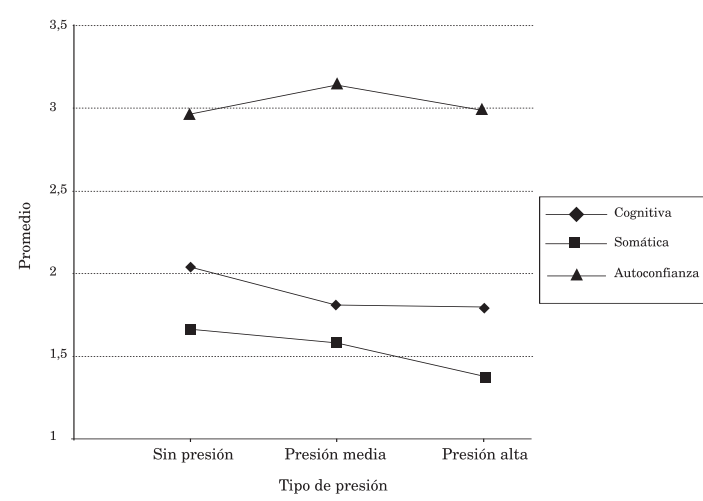

El Gráfico No. 8 muestra que la tensión medida con el cuestionario POMS, tiende de modo muy similar, a descender de la primera a la tercera medición en ambos grupos, pero que siempre es menor en los avanzados que en los principiantes. La caída más brusca se da en los avanzados entre la segunda y tercera medición. 
Gráfico No. 8

Tensión por grupo según el tipo de presión

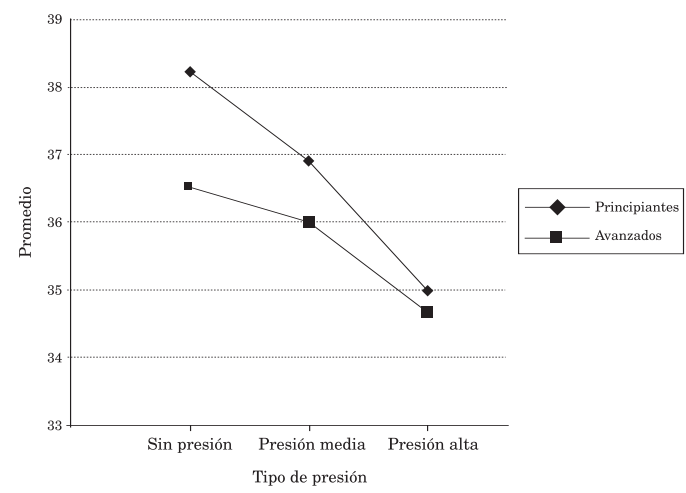

Mediante la aplicación del un ANOVA de medidas repetidas a cada grupo se determinaron los efectos de la presión alta, media y baja, causaba diferencias significativas en cada variable medida.

En el Cuadro No. 3 se anotan los resultados de ese análisis. De este modo es posible observar que la mayor parte de las diferencias se presentan entre los sujetos del grupo de avanzados y el menor número de estas se dan entre los intentos (efectividad y tensión).

En cuanto al grupo de principiantes presenta diferencias entre los sujetos en la frecuencia cardiaca y la tensión en tanto que todas las variables son significativas en los sujetos del grupo de avanzados.
Sin embargo resulta de mayor interés para este estudio el que las diferencias entre los intentos (según tipo de presión), se presentan en los principiantes sólo en la efectividad con 3.68 y la tensión con 3.98, para una $\mathrm{Ft}(2,16)$ $\mathrm{p}<.05: 3.63$.

La única diferencia entre los intentos (tipo de presión) de los avanzados se presenta en la efectividad con 5.57 para una $\mathrm{Ft}_{(2,16)} \mathrm{p}<.05: 3.63$

En resumen el tipo de presión afecta significativamente los resultados de la efectividad y la tensión en los principiantes y sólo la efectividad en los avanzados.

El análisis post hoc mediante la aplicación de la prueba de Newmans Keuls, permite afirmar que en la efectividad de los principiantes, la significancia se establece entre la medición con presión media y la de presión alta con una $\mathrm{f}_{\mathrm{c}}$ de 3 contra la $\mathrm{ft}_{(2,16)} \mathrm{p}<.05: 3$ y el segundo resultado se presenta entre la medición sin presión y presión alta con una f calculada de 3.63 que iguala la de la tabla a 3 y 16 grados de libertad para un nivel de $\mathrm{p}<.05$.

En el grupo de avanzados se concentra toda entre los intentos sin presión y presión alta. El post hoc asigna un valor calculado de 4.05 contra uno tabular de 3.61 con 3 y 18 grados de libertad a un nivel de $\mathrm{p}<.05$., así la diferencia entre los

Cuadro No. 3

Resumen del ANOVA de medidas repetidas por grupo y tipo de presión en cada variable deportiva y psicológica

\begin{tabular}{|c|c|c|c|c|}
\hline & \multicolumn{2}{|c|}{ Principiantes } & \multicolumn{2}{|c|}{ Avanzados } \\
\hline & F sujetos & $\mathrm{F}$ intentos & F sujetos & $\mathrm{F}$ intentos \\
\hline Efectividad & 2.28 & $3.68^{*}$ & $3.99 *$ & $5.57 *$ \\
\hline Ansiedad cognitiva & 1.23 & .44 & $3.53^{*}$ & 1.93 \\
\hline Ansiedad somática & .11 & .84 & $5.59 *$ & 2.37 \\
\hline Autoconfianza & 1.70 & .59 & $4.79 *$ & .78 \\
\hline Frecuencia cardiaca & 3. $66^{*}$ & 1.27 & $5.88^{*}$ & .01 \\
\hline Tensión & $12.47^{*}$ & $3.98^{*}$ & $3.27 *$ & .70 \\
\hline
\end{tabular}

Ft $(8,16) \mathrm{p}<. .05: 2.59 \mathrm{Ft}(2,16) \mathrm{p}<. .05: 3.63 \quad \mathrm{Ft}(8,16) \mathrm{p}<. .05: 2.59 \quad \mathrm{Ft}(2,16) \mathrm{p}<. .05: 3.63$ 
intentos de los principiantes se reparte en dos niveles en tanto que en los avanzados sólo se refleja en uno según el tipo de presión.

Otra variable que reflejó una diferencia significativa entre los intentos o tipo de presión, fue la tensión y sólo en el grupo de principiantes. El post-hoc permite asegurar que la diferencia se concentró entre la medición sin presión y con presión alta, la cual muestra una $\mathrm{f}_{\mathrm{c}}$ de 3.98 contra la f de la tabla que es de 3.65 con 3 y 16 grados de libertad a un nivel de $\mathrm{p}<.05$.

Por otra parte, el análisis de los Tamaños del Efecto, de la presión sobre cada una de las variables y los resultados para el grupo de los principiantes, reportan efectos altos entre la primera y segunda medición (sin presión-presión media) en la efectividad -.95 en tanto que se presentan tamaños moderados en la ansiedad somática: .68.

Entre la medición de presión media y presión alta sólo se reporta tamaños del efecto muy alto de .82, en la efectividad. El análisis sin presión y presión alta no reporta ningún tamaño del efecto alto sino más bien dos moderados con .58 y .62 en la ansiedad cognitiva y la tensión respectivamente. El total de resultados se resumen en el Cuadro No. 4.

En cuanto a los avanzados, se observan Tamaños del Efecto altos entre la primera y segunda medición en la ansiedad somática, 1.53 entre la primera y tercera medición en la efectividad .77.

Los efectos moderados se presentan sólo entre la primera y segunda medición en la ansiedad cognitiva y en la ansiedad somática entre la primera y tercera medición. Los resultados se resumen en el Cuadro No. 5 .

Cuadro No. 4

Resultados de las pruebas del tamaño del efecto en los principiantes según el tipo de presión

\begin{tabular}{lccc}
\hline & $\begin{array}{c}\text { Sin presión - presión } \\
\text { media }\end{array}$ & $\begin{array}{c}\text { Presión media - } \\
\text { presión alta }\end{array}$ & $\begin{array}{c}\text { Sin presión - presión } \\
\text { alta }\end{array}$ \\
\hline Efectividad & $.95^{*}$ & $.82^{*}$ & -.18 \\
Ansiedad Cognitiva & .12 & .53 & .58 \\
Ansiedad Somática & $.68^{*}$ & -.07 & $-.58^{*}$ \\
Autoconfianza & .44 & .07 & .41 \\
Frecuencia cardíaca & .14 & -.17 & .37 \\
Tensión & .26 & .30 & $-.62^{*}$ \\
\hline
\end{tabular}

$* \mathrm{p} \leq 0.05$

Cuadro No. 5

Resultados de las pruebas del tamaño del efecto en los avanzados según el tipo de presión

\begin{tabular}{lccc}
\hline & $\begin{array}{c}\text { Sin presión - presión } \\
\text { media }\end{array}$ & $\begin{array}{c}\text { Presión media - } \\
\text { presión alta }\end{array}$ & $\begin{array}{c}\text { Sin presión - presión } \\
\text { alta }\end{array}$ \\
\hline Efectividad & .34 & .49 & $.77^{*}$ \\
Ansiedad cognitiva & $.60^{*}$ & .25 & .54 \\
Ansiedad somática & $1.53^{*}$ & .05 & $.60^{*}$ \\
Auotconfianza & -.032 & .34 & -.04 \\
Frecuencia cardíaca & 0 & -.03 & -.03 \\
Tensión & .12 & .33 & .43 \\
\hline
\end{tabular}


Las diferencias en cada una de las mediciones y variables se observan en los gráficos que van del 1 al 5 y el 8, en los que aparecen las tres mediciones de cada grupo en cada variable.

Mediante un Anova Factorial Mixto interesó determinar las diferencias entre los grupos, (asociado al nivel de destreza), entre las mediciones correspondientes a niveles de presión, (sin presión, presión media y presión alta) y la interacción de la presión de los grupos. (Cuadro No. 6).

Los resultados de este análisis aportan interacciones significativas en la efectividad: 6.32 comparada contra una $\mathrm{f}$ tabular de 4.45 a p <.05 y la autoconfianza y la frecuencia cardiaca entre los grupos con resultados de $6.97 \mathrm{p}<.01$ en la primera $\mathrm{y}$ $8.18 \mathrm{p}<.006$ en la segunda.

La única diferencia entre las mediciones o tipo de presión en los grupos se reporta en la efectividad 12.26 contra una $f$ tabular de 5.29 a p $\geq .001$.

Sin embargo, quizá lo que más interesa destacar es la interacción que se da entre la efectividad de los grupos y mediciones bajo diferentes condiciones de presión, cuya f calculada es altamente significativa 15.73 para una $\mathrm{ft}_{(2,34)}$ $5.29 \mathrm{p} \geq .001$.

La aplicación de la prueba post hoc de Newmans Keuls permitió determinar que la interacción se justificaba básicamente por los resultados de las mediciones de avanzados sin presión contra la de los principiantes sin presión cuya f tabular fue de 13.43 la cual resultó significativa al compararla con $\mathrm{ft}_{(2,6)} \mathrm{p}<.05$ : 11.7. El otro resultado de interacción significativa se presentó entre los avanzados sin presión y los principiantes con presión alta cuya f de 12 resultó significativa ante la $\mathrm{ft}_{(2,5)} \mathrm{p}<.05: 10.9$. En resumen la interacción de las mediciones de los grupos según el tipo de presión justifica por las dos significancias citadas anteriormente.

Finalmente, el análisis de las correlaciones en cada uno de los grupos según el tipo de presión para determinar el valor de predicción de cada variable psicológica sobre la efectividad, refleja correlaciones significativas solo para el grupo de principiantes bajo presión media a $\mathrm{p}<.01$ en la variable frecuencia cardiaca con .76. Los avanzados no reportan ninguna correlación significativa.

El análisis estadístico de regresión múltiple no refleja ninguna relación significativa entre todas las variables con respecto a la efectividad.

El análisis de regresión múltiple no presentó relaciones significativas en ninguna de las escalas correspondientes a la medición de la ansiedad, aunque si se presentan algunas significancias en la hostilidad, la depresión y la confusión del instrumento POMS, pero las mismas no interesan para efectos de este estudio.

Cuadro No. 6

Resumen del ANOVA factorial mixta entre grupos (nivel de destreza) por variables físicas, psicológicas y tipo de presión (medición)

\begin{tabular}{lrlrlrl}
\hline & \multicolumn{2}{c}{ Grupo } & \multicolumn{2}{c}{ Presión } & \multicolumn{2}{c}{ Interacción } \\
\hline Efectividad & 6.32 & $(\mathrm{p} \leq .05)^{*}$ & 12.26 & $(\mathrm{p} \leq .001)^{* *}$ & $15.73 \quad(\mathrm{p} \leq .001)^{* *}$ \\
Ansiedad cognitiva & 2.11 & $(\mathrm{p} \leq 1.52)$ & 1.66 & $(\mathrm{p} \leq .20)$ & $.30 \quad(\mathrm{p} \leq .74)$ \\
Ansiedad somática & 3.27 & $(\mathrm{p} \leq .07)$ & 1.58 & $(\mathrm{p} \leq .21)$ & $.38 \quad(\mathrm{p} \leq .38)$ \\
Autoconfianza & 6.97 & $(\mathrm{p} \leq .01)^{* *}$ & .22 & $(\mathrm{p} \leq .80)$ & $.60 \quad(\mathrm{p} \leq .68)$ \\
Frecuencia cardíaca & 8.18 & $(\mathrm{p} \leq .006)^{* *}$ & .23 & $(\mathrm{p} \leq .80)$ & $.36 \quad(\mathrm{p} \leq .55)$ \\
Tensión & .64 & $(\mathrm{p} \leq .43)$ & 1.19 & $(\mathrm{p} \leq .31)$ & $1.46 \quad(\mathrm{p} \leq .86)$ \\
\hline
\end{tabular}




\section{Discusión}

Las relaciones establecidas en las investigaciones con respecto a la ejecución óptima y los niveles de ansiedad si bien muestran tendencias grupales, estas no son definitivas, pues a partir de las curvas individuales, no puede afirmarse categóricamente que un nivel mayor de tensión y ansiedad se asocie con niveles mayores de rendimiento o inversamente, como tampoco puede afirmarse, que la ejecución siempre mejora con el aumento de ansiedad y que a partir de cierto límite, empieza a decrecer aunque la ansiedad continúe en ascenso.

Las investigaciones han mostrado que hay diferencias individuales en relación con la ansiedad las cuales no se reflejan en el comportamiento grupal. (Gill 1994, Maynard y otros 1995, Gould y Krane 1992).

Los resultados del ANOVA de una vía para cada grupo, especialmente en el de avanzados, demuestra la existencia de diferencias significativas entre los sujetos en todas las variables psicológicas y la efectividad. En el grupo de principiantes las significancias aparecen sólo en la frecuencia cardíaca y la tensión y los mismos muestran tantas curvas diferentes como sujetos hay en cada grupo. Sin embargo, uno de los resultados de más relevancia, en el Anova de dos vías, tal vez sea el relacionado con la interacción significativa que se presenta en la efectividad. Así, es evidente que la relación del rendimiento en la efectividad depende del nivel de destreza de los ejecutantes y de la presión, este resultado guarda relación con las diferencias obtenidas en cada grupo al realizar las tres mediciones y las halladas ente los sujetos del grupo de avanzados, lo cual podría explicarse posiblemente por la especialización en el juego, en tanto que en los principiantes este elemento no está presente.

La presión ejercida en la ejecución afectó significativamente a ambos grupos independientemente de su grado de des- treza y experiencia con lo cual se puede sostener que la experiencia bajo diferentes condiciones de presión no va a determinar un manejo más adecuado de la eficacia de la destreza. Los patinadores de élite con los cuales trabajó Gould (1992), en su investigación para determinar las fuentes de ansiedad, manifestaron que la ansiedad es mayor cuanto más alto sea el nivel de competencia, especialmente debido a que se debe seguir demostrando por qué se está en los primeros lugares de las competencias y cada vez sus resultados deben ser mejores.

Por lo tanto se estima que sería conveniente determinar qué factores o fuentes de presión son los que intervienen en los resultados de los jugadores de baloncesto nacionales, bajo condiciones de competencia o rendimiento específico.

Así, a partir de los resultados obtenidos y otras investigaciones, es posible también afirmar, que la ansiedad como una respuesta multidimensional a la tensión tiene un carácter individual, siendo importante destacar, que la aplicación de programas de contención de la ansiedad o de activación psico-motriz, deben de planificarse y desarrollarse a partir de las diferencias individuales aunque en el caso de deportes de conjunto deben orientarse hacia el logro de los objetivos grupales. Williams y Sperryn (1982), recomiendan que debido a las diferencias individuales de rendimiento y personalidad, asociadas a necesidades y respuestas psíquicas diferentes, la atención de los sujetos debe ser absolutamente personalizada. El análisis de los gráficos de la efectividad de cada sujeto según el tipo de presión, demuestra que no existe un patrón único de respuestas dada la condición de principiantes o avanzados, ni en la ejecución ni en los valores de la ansiedad, aunque sí podrían inferirse algunas tendencias.

Se halló que el 47,5\% de los principiantes contra un $30 \%$ de los avanzados, muestran un patrón de U invertida. Otro $30 \%$ de los avanzados se agrupan con un patrón de U normal, lo cual sumado al $30 \%$ 
de U invertida sustenta el hecho de que hay una tendencia en este grupo a conductas más homogéneas en el rendimiento en comparación con el grupo de principiantes, pues en este último grupo, el $43,5 \%$ se reparte en cantidades iguales $(9,5 \%)$ entre curvas ascendentes a la derecha, lineales y combinadas (lineal ascendente y lineal descendente), en tanto que el grupo de los avanzados un $20 \%$ se agrupa en patrón lineal descendente a la derecha, un $10 \%$ con un modelo lineal y un $10 \%$ con uno combinado lineal descendente.

Es posible que este comportamiento se halle relacionado con el nivel de dominio de la destreza, ya que es de esperar que sujetos que conforman un equipo que participa formalmente en torneos nacionales sean lo más homogéneos posible en la ejecución técnica.

Sin embargo, es importante afirmar que la tendencia mayoritaria manifestada por los principiantes, de patrón de rendimiento de $\mathrm{U}$ invertida, se refleja en el diseño gráfico grupal, en tanto que en el grupo de avanzados, posiblemente debido a que se hallan más repartidos en dos tendencias la curva grupal se muestra descendente hacia la derecha. (Gráfico 1). Así, se puede afirmar que conforme aumenta la presión el grupo de sujetos avanzados tendió a realizar ejecuciones menos efectivas en tanto que en los principiantes se observa una relación ascendente del rendimiento y la presión hasta cierto punto y después, a pesar de que la presión se aumenta, se manifiesta un descenso en el rendimiento.

Podría establecerse algún tipo de relación entre estas curvas observadas y el grado de ansiedad de cada sujeto de acuerdo con las teorías que diferentes autores plantean con respecto a las respuestas de ansiedad en relación con el rendimiento, lo cual quedará para estudios posteriores. Sin embargo, puede reportarse que al analizar las curvas individuales de la ansiedad en cada uno de los componentes que contiene el CSAI-2: cognitivo, somático y auto- confianza, así como la tensión del POMS, se hallaron resultados similares al de la efectividad, lo cual permite comprobar aun más que la ansiedad tiene características de absoluta inherencia a cada sujeto, y que por lo tanto las decisiones para la intervención debe hacerse sobre esta base, aunque a partir de los análisis pueden existir tendencias generales de carácter grupal que también deben ser consideradas.

Lo anterior permitió apoyar el hecho de que, para este caso específico no puede hablarse de absolutos en cuanto a la relación rendimiento deportivo, presión ambiental.

Se asume que los jugadores de más experiencia están más habituados a rendir bajo condiciones de más presión que aquellos que no lo son, sin embargo, los resultados demostraron que es a niveles menores de presión que estos realizan sus mejores ejecuciones.

A partir de la observación de las curvas de rendimiento grupal, se concluyen claras diferencias en las mismas, las cuales no parecen depender sólo del grado de destreza de los sujetos sino de su activación ante el reto que tendrían que enfrentar, y contrariamente a lo que se ha apuntado, respecto a que los sujetos más experimentados exhiben menores grados de ansiedad y pueden lograr un mayor nivel de ejecución. En este caso llama la atención, que más bien es con menor presión que los avanzados logran su mayor rendimiento en tanto que los principiantes lo hacen con la presión intermedia.

La revisión de literatura apoya el hecho de que uno de los elementos que determina la relación de ansiedad-ejecución es el referente a la complejidad de la tarea. Wrisberg (1994), señala específicamente, que en el baloncesto parece ser necesario un nivel mayor de activación psicológica en aspectos relacionados con la defensa que para los del tiro libre, lo cual parece corroborarse en este caso cuando los avanzados mostraron un rendimiento mayor, 
(X: 5,20), en aquella condición sin presión que en los niveles de presión media (X: 4,50), o alta, (X: 3), contrariamente a los principiantes, quienes bajo presión media, logran mejores resultados, (X: 4), sin embargo, siempre son mejores en promedio los del grupo de avanzados, explicado ello por el grado de dominio de la misma.

Los resultados obtenidos permiten continuar apoyando los resultados obtenidos en otras investigaciones en los últimos años respecto a que la ansiedad es una respuesta multidimensional que varía de un sujeto a otro, lo cual a su vez igualmente se corrobora a nivel grupal ( Gill, 1994) además de que, como afirma Wrisbeg (1994) no puede a pesar de los resultados, pensarse que existe un patrón único válido para todas las situaciones, aun en condiciones similares, especialmente porque existe una serie de elementos ajenos a la prueba o competencia de carácter interno o externo que van a estar afectando la relación ansiedad-ejecución. (Lazarus, 1986).

Con base en lo hallado, no es recomendable suponer que determinado grupo de deportistas va a mostrar o no, similitud en las respuestas, ello va a estar relacionado con cada sujeto, su percepción de condiciones externas y afrontamiento de las internas, todo lo cual determinará su activación ante el reto y las respuestas motrices y psicológicas, lo cual conduce a recomendar el análisis de los grupos en el contexto específico.

Recuérdese que las últimas investigaciones en este campo plantean justamente la importancia que tiene determinar cuál es la zona de rendimiento adecuada a cada sujeto bajo diferentes condiciones de ejecución, lo que redundará en los beneficios personales y grupales cuando sea el caso, a partir de la planificación de un trabajo físico y psicológico orientado al desarrollo y fortalecimiento de los elementos necesarios para que cada individuo pueda alcanzar esa zona de rendimiento óptimo. (Gordin y Reardon 1995, Hannin 1995). Sin embargo no siendo el objetivo de este estudio determinar cuáles son esas zonas de rendimiento óptimo para cada sujeto, no fue considerado ese aspecto, siendo una recomendación importante el considerarlo para un próximo estudio.

Además se estima también importante realizar un estudio en el que se mida la ansiedad posterior a la ejecución de la prueba a fin de determinar los cambios que se presentan en esta, producto de la manipulación ambiental. De igual modo puede resultar interesante, y con base en lo que apoya la literatura, realizar pruebas de destreza como esta y otras de que impliquen situación de juego de ataque, defensa o una combinación de ambas, lo cual permitiría comprobar si se apoya lo que Wrisberg (1994) propone respecto a las destrezas en relación con su complejidad, calidad de cerradas o abiertas o bien su característica de destreza cíclica o acíclica.

\section{Referencias bibliográficas}

Campbell, D. y Stanley, J. Diseños experimentales y cuasi experimentales en la investigación social. Argentina, Amorrotu Editores, 1978.

Delgado S. G., "Influencia de la ansiedad en el rendimiento deportivo de los futbolistas de la I División en Costa Rica”, Memoria del II Simposio Internacional en Ciencia del movimiento Humano, Universidad de Costa Rica, San José, Costa Rica, 26 Octubre 1995.

Delgado S. G., "La ansiedad de los dirigentes, aficionados, y no aficionados en relación con el tiempo, y el ambiente de un equipo de futbol de la I División en Costa Rica”, Memoria del III 
Simposio Internacional en Ciencias del Movimiento Humano, Universidad de Costa Rica, San José, Costa Rica, 2-4 Octubre 1996.

Gill, D. "An sport an exercise psychology perspective on stress". Quest, 46 (1), USA, 1994.

Gordin, R. D, y Reardon, J. P. "Achieving the zone: the study of flow in sport" Sport psychology: an analysis of athlete behavior, Massachusetts, USA, Movement Publications, 1995.

Gould, D. y Krane, V. "The arousal athletic performance relationship: current status and future directions", Advances in sport psychology, chapter 6, Champaign, Illinois, USA, Human Kinetics, 1992.

Hanin, Y.L. "Individual zones functioning." Sport psychology: an analysis of athlete behavior. Massachusetts: Movement Publications, 1995.
Kerlinger, F.N, Investigación del comportamiento, México: Mac-Graw Hill Editores, 1988.

McNair, D., Lorr, M. y Droppleman, L.F. EITS Manual for the profile of mood states. San Diego, California: Educational and Industrial Testing Service, 1971 .

Martens, R., Vaeley, R., y Burton, D. “Competitive anxiety in sport”, Champaign, Illinois, USA, Human Kinetics,1990.

Unesthal L E. L. "Ideal performance”, Sport psychology: an analysis of athlete behavior. Massachusetts, Illinois, USA, Movement Publications, 1995.

Williams, J .G. P. y Sperryn, P.N. S. Medicina deportiva. Barcelona, España: Salvat Editores S.A, 1982.

Wrisberg, C. A, “The arousal-perfomance relationship”, Quest, 46 (1), USA, 1994.

Guadalupe Delgado Socatelli Profesora de la Escuela de Educación

Física y Deportes Universidad de Costa Rica 